\title{
Lipid related consequences of intestinal malabsorption
}

\author{
GILBERT R THOMPSON
}

SUMmary The small intestine plays a key role in lipid metabolism by absorbing fat and synthesising apoproteins. Fat malabsorption secondary to intestinal disease results in abnormalities of lipoprotein concentration and composition and can lead to deficiency of essential fatty acids and fat-soluble vitamins. Malabsorption of fat can be induced by administration of neomycin and malabsorption of bile acids by administration of anion-exchange resins or by creating a partial ileal bypass. These induced forms of malabsorption are useful in the treatment of hyperlipidaemic patients liable to atherosclerosis.

This review spans 25 years of research in the field of lipids. During that time major advances in knowledge and changes in attitude have taken place, culminating in the award of the Nobel Prize to Brown and Goldstein in 1985 for their discovery of the low density lipoprotein (LDL) receptor. My own interest in the subject was awakened while working as a registrar with Chris Booth at the Hammersmith and seeing a patient of his with steatorrhoea and severe osteomalacia. This led me to study vitamin D absorption and subsequently, while working with Kurt Isselbacher in Boston, I became interested in that closely related compound, cholesterol. On returning to the Hammersmith I studied the lipoprotein abnormalities which accompany malabsorption syndromes and the therapeutic potential of induced malabsorption in hyperlipidaemic patients. Thus my lipophilic interests have taken me from the intestinal lumen to the arterial wall, a logical but initially uphill journey. I am grateful to Chris Booth for giving me the freedom to pursue my own research interests and also to those colleagues who assisted my endeavours, whose work is reviewed in this tribute to him.

\section{Absorption of dietary fat and fat soluble vitamins}

Lipid absorption can be depicted as occurring in four separate stages, as follows: (1) An intraluminal phase of lipolysis and micellar solubilisation. (2) A mucosal phase comprising the uptake of micellar lipids and, in most instances, their subsequent re-esterification. (3) A lymphatic phase, which involves chylomicron formation and secretion into lymph. (4) A phase of intravascular metabolism, encompassing the hydrolysis of chylomicrons in peripheral blood vessels, the uptake of their remnants by the liver and the subsequent recycling and exchange of lipids and apoproteins between the various lipoproteins present in plasma.
INTRAL.UMINAL. PHASE

The intraluminal phase is dependent upon the presence of adequate concentrations of pancreatic lipase to hydrolyse triglyceride into monoglyceride and fatty acid, and of bile salts to incorporate these products of lipolysis into mixed micelles. In addition, non-polar sterols such as cholesterol and fat soluble vitamins must be incorporated into the core of mixed micelles as a prerequisite to their absorption. The efficient uptake of monoglyceride and, to a lesser extent, of cholesterol by the jejunum contrasts with that of bile salts, which are not absorbed until the ileum.

The importance of bile salts in the absorption of fat soluble vitamins has been known for many years. Borgström showed that during fat absorption the intestinal contents could be separated by ultracentrifugation into a supernatant oil phase and an infranatant aqueous, or micellar, phase. Subsequently, Hofmann and he showed that the micellar phase consisted of aggregates, $5 \mathrm{~m} \mu$ in diameter, of monoglycerides, ionised fatty acids and bile salts, termed mixed micelles whereas the oil phase consisted mainly of unhydrolysed triglyceride in the form of emulsion particles, $1,000 \mathrm{~m} \mu$ in diameter. Nonpolar lipids such as cholesterol and the fat-soluble vitamins partition themselves between the oil and micellar phases. There is some evidence that polar lipids, such as fatty acids, can be absorbed reasonably well from emulsions and that incorporation into mixed micelles is not a prerequisite for their absorption. In the case of non-polar lipids, however, micelle formation is essential.

MUCOSAL PHASE

Electron microscopic studies show the presence of osmiophilic droplets in the region of the Golgi and in the intracellular spaces during the mucosal phase of absorption. These appearances reflect the resynthe- 
sis of triglyceride from absorbed fatty acid and monoglyceride, its subsequent incorporation into chylomicrons and their extrusion from the cell by reverse pinocytosis.

After uptake as the free sterol, cholesterol is largely re-esterified during its passage through the intestinal mucosa. Approximately two thirds of the absorbed sterol undergoes esterification by acyl cholesterol acyl transferase (ACAT), mainly to cholesterol oleate. Vitamin A (retinol) is also largely re-esterified during absorption, preferentially with palmitic acid, but vitamins $D, E$ and $K$ pass through the intestinal mucosa without major modifications.

LYMPHATIC PHASE

During this phase of absorption numerous chylomicrons can be seen within intestinal lymphatics. The major route of transport of triglycerides, cholesterol and the fat soluble vitamins from the intestinal mucosa into blood is via the lymph. The rate of exit of free cholesterol and vitamin D into lymph is increased by the simultaneous administration of exogenous triglyceride ${ }^{3}$ and vitamin $\mathrm{E}$ absorption is stimulated in a similar manner. ${ }^{+}$The enhanced absorption is probably related to the increased synthesis of chylomicrons that occurs during fat absorption, within which cholesterol and the fat soluble vitamins are largely transported. Green and Glickman ${ }^{5}$ have shown that the small intestine synthesises several of the apoproteins which occur in chylomicrons, including apoB48, apoA-IV and apoA-I, and $\mathrm{Wu}$ and Windmueller have shown that over $50 \%$ of the apoA-I and apoA-IV come from the small intestine.

Although intestinal lymph is undoubtedly the major pathway of absorption for cholesterol and the fat soluble vitamins, it is probable that some absorption also occurs via the portal vein. Evidence for this has been put forward for vitamin $\mathrm{E}$ by MacMahon et al, ${ }^{4}$ who found higher concentrations of labelled $\alpha$-tocopherol in portal venous than in aortic plasma after oral administration. This presumably explains why small amounts of vitamin E can be demonstrated in the blood of treated patients with a $\beta$-lipoproteinaemia, in whom lymphatic transport of lipids cannot occur.

INTRAVASCULAR METABOLISM

Chylomicrons mix with HDL in lymph, pick up apoE and $\mathrm{C}$, and then get partially degraded by lipoprotein lipase. The resulting chylomicron remnants are quickly taken up by chylomicron remnant receptors in the liver. It is thought that this process is mediated by specific receptors which recognise the apoE on the surface of remnant particles but are metabolically distinct from LDL receptors which recognise
apoB 100 as well as apoE. During lipolysis, some of the free cholesterol and lecithin on the surface of chylomicrons is released into plasma and this coalesces with nascent $\mathrm{HDL}_{3}$ particles which have been secreted by the liver and the small intestine. As a result of the action of lecithin:cholesterol acyltransferase (LCAT) the free cholesterol gets esterified to cholesterol ester and the particle enlarges and becomes $\mathrm{HDL}_{2}$. Very low density lipoprotein (VLDL) containing apoB100, is synthesised predominantly in the liver but to some extent in the small intestine. This too gets hydrolysed by the action of lipoprotein lipase to form VLDL remnants. The latter are then either taken up by the LDL receptor or are acted upon by hepatic lipase to give rise to LDL. Low density lipoprotein circulates in plasma and eventually gets taken up by LDL receptors located both in peripheral cells and in the liver.

\section{Consequences of acquired malabsorption}

Malabsorption can be secondary to intraluminal dysfunction, as in pnacreatic insufficiency, inflammatory bowel disease and intestinal resection or it can be associated with mucosal dysfunction, as found in coeliac disease, tropical sprue, and after the administration of large doses of neomycin. It can also be caused by lymphatic dysfunction, as seen in intestinal lymphangiectasia, Whipple's disease and filariasis. Lastly, it can be secondary to manoeuvres which stimulates bile acid excretion, such as the administration of anion-exchange resins or the creation of a partial ileal bypass.

\section{FAT SOLUBLE VITAMIN DEFICIENCIES}

There are numerous case reports of patients with malabsorptive disorders developing clinical deficiency of vitamins D or K. Biochemical evidence of vitamin E deficiency has also been demonstrated quite frequently in such patients, although its clinical significance is difficult to assess. ${ }^{\text {? }}$

In view of the importance of micelle formation for the absorption of non-polar lipids it is not surprising that severe malabsorption of labelled vitamins $A, D$, $E$ and $K_{1}$ has been shown in patients with biliary obstruction in whom cannulation of the thoracic duct had been undertaken. Other studies, in which the faecal excretion of radioactivity was used as an index of absorption, showed marked malabsorption of both labelled vitamins $D^{\times}$and $E^{7}$ in patients with steatorrhoea of biliary or pancreatic origin. Fat soluble vitamin deficiencies can also occur after partial gastrectomy or small intestinal resection. Vitamin D absorption is usually only slightly subnormal in patients with postgastrectomy steatorrhoea," however, and may even be normal, suggesting that lack 


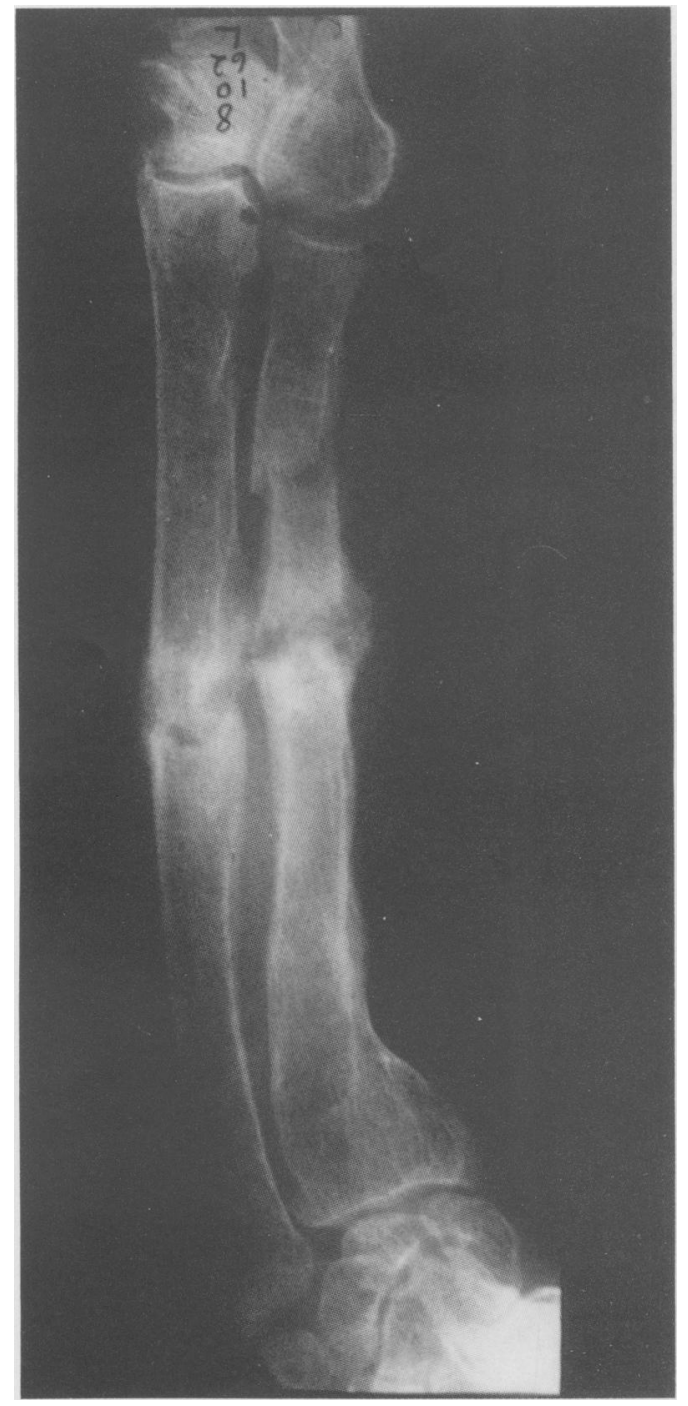

Fig. 1 Pathological fractures (Looser's zones) in radius and ulna of 66 year old woman $(G H)$ with osteomalacia and steatorrhoea. (Reproduced with permission from ref 11).

of sunlight or dietary deficiency is a more important factor in causing their osteomalacia." In contrast, patients with adult coeliac disease often show marked malabsorption of vitamin $\mathrm{D}$, the severity being directly proportional to the degree of steatorrhoea. ${ }^{8}$ Studies on the absorption of vitamin E showed marked malabsorption in two patients with intestinal lymphangiectasia.?

A particularly striking case of osteomalacia, referred to in the Introduction, was associated with megaloblastic anaemia and steatorrhoea secondary

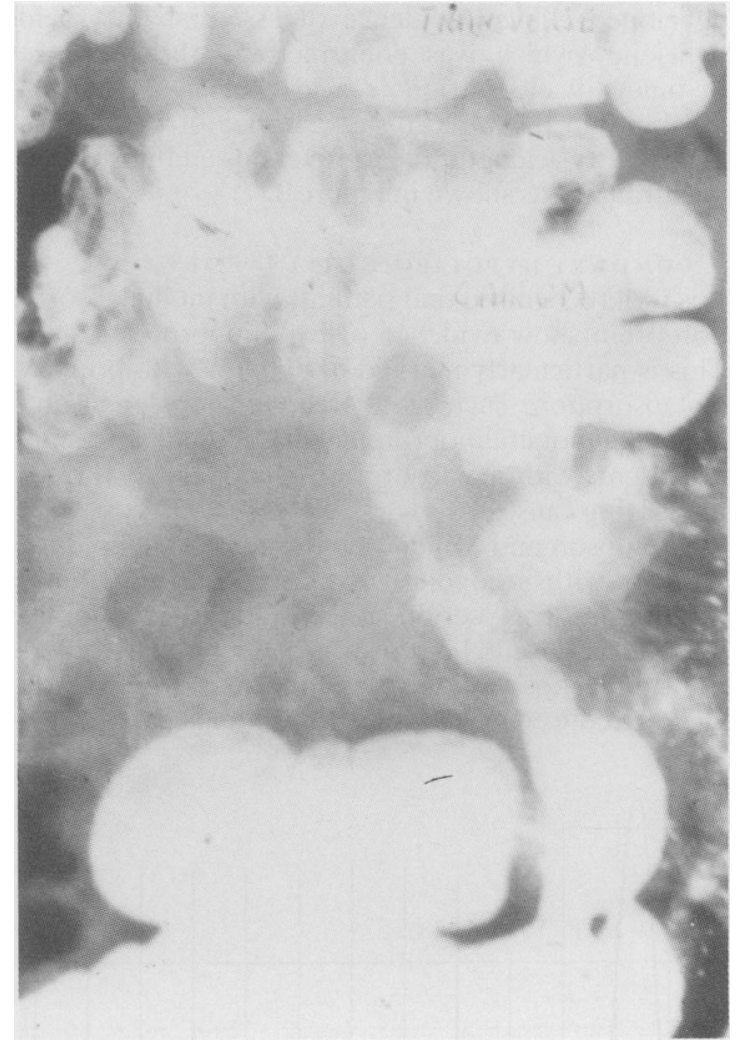

Fig 2 Barium follow through showing stricture of terminal ileum and anomalously situated caecum and ascending colon in $\mathrm{GH}$. (Reproduced with permission from ref. 11).

to a stricture of the terminal ileum, " as illustrated in Figures 1 and 2. It seems probable that the ileal lesion was responsible not only for malabsorption of vitamin $B_{12}$ but also of bile acids, thereby impairing micelle formation and causing marked malabsorption of vitamin $\mathrm{D}$, comparable with that demonstrated in rats fed cholestyramine. ${ }^{12}$

\section{ESSENTIAL FATTY ACID DEFICIENCY}

Shimoyama et al $^{13}$ examined the fatty acid composition of plasma lipids in patients with malabsorption and showed that there was a decrease in the proportion of linoleic acid (18:2) and arachidonic acid (20:4) in malabsorbers compared with healthy controls and patients without malabsorption. These changes were most marked in the fatty acid composition of cholesterol esters and lecithin.

When various categories of malabsorption were examined it was found that the decrease in essential fatty acids was most marked in patients who had an intestinal resection. Subsequently, Press et $^{\text {al }}{ }^{14}$ described three patients with intestinal resection who 
developed clinical evidence of essential fatty acid deficiency which was confirmed by the presence of omega-9 cicosatrienoic acid (20:3 $\omega 9$ in plasma lecithin and the rapid disappearance of this pathological fatty acid after the intravenous administration of Intralipid, as shown in Figure 3.

\section{SECONDARY HYPOLIPOPROTEINAEMIA}

It is well recognised that patients with fat malabsorption often show evidence of hypolipoproteinaemia. This is particularly marked in patients with primary malabsorption, such as abetalipoproteinaemia and familial hypobetalipoproteinaemia, but it also occurs in patients with malabsorption secondary to various underlying causes.

Thompson and Miller ${ }^{15}$ measured plasma lipids in patients with secondary malabsorption and found that plasma triglyceride concentrations were either normal or raised. In contrast, their plasma cholesterol and phospholipid concentrations were nearly always reduced. Quantification of plasma lipopro- teins by ultracentrifugation showed that VLDL concentrations were either normal or raised, but LDL concentrations tended to be reduced, especially in men, whereas in women there was a more marked reduction in HDL, as shown in Figure 4. Press and Thompson ${ }^{16}$ measured plasma postheparin lipolytic activity in some of these patients and showed that the activity of lipoprotein lipase was markedly reduced, which provided an explanation for the increased levels of VLDL and triglyceride.

In addition to noticing that the concentration of LDL was reduced, Thompson and Miller ${ }^{15}$ showed that its composition was abnormal, in that there was a considerable reduction in the percentage of cholesterol ester in the LDL of malabsorbers and an increase in the percentage of triglyceride, these changes being inversely correlated. Miller and Thompson ${ }^{17}$ then showed that LCAT activity in the plasma of malabsorbers was reduced and suggested that this was responsible for the decrease in the cholesterol ester content of their LDL.
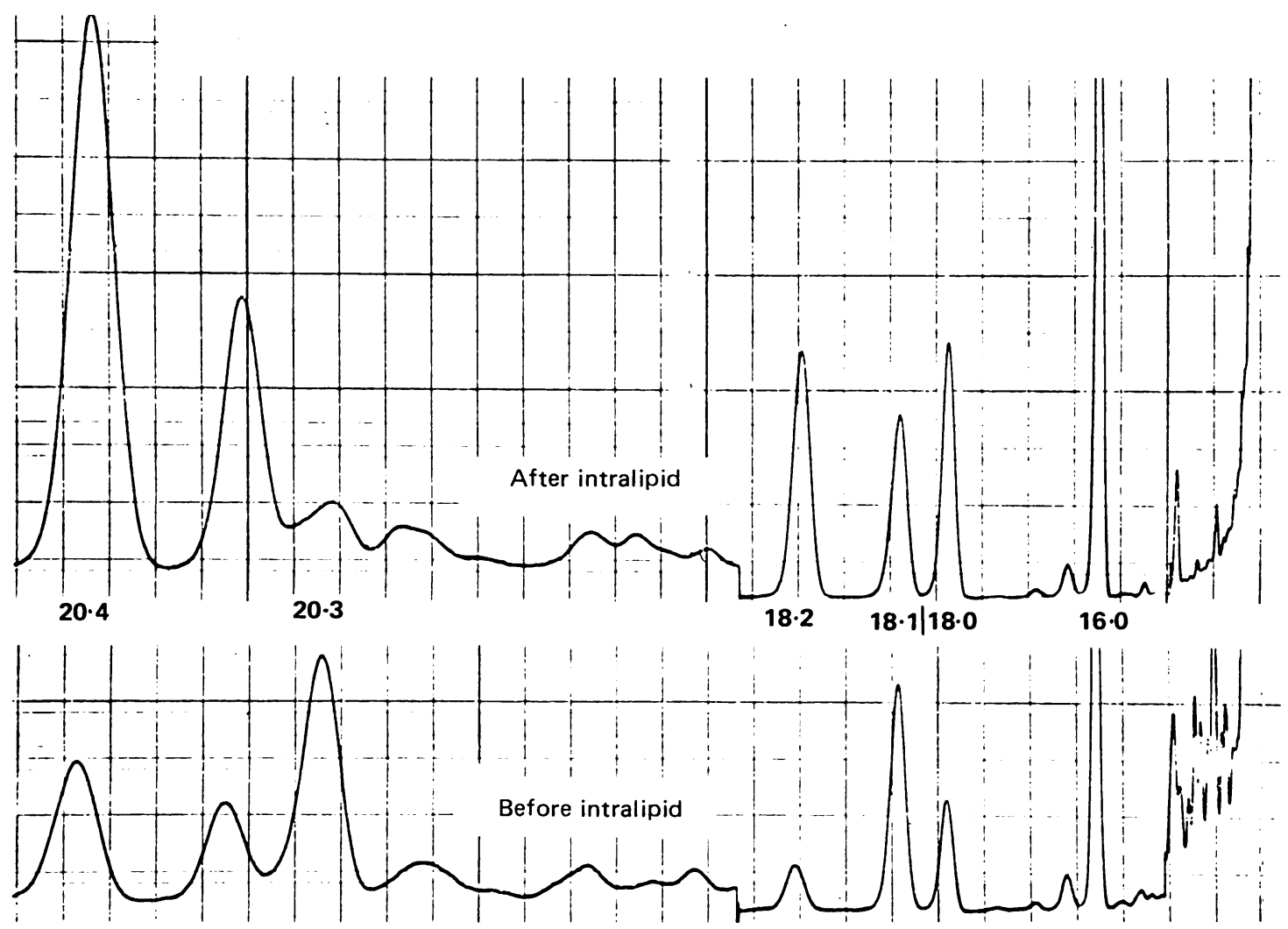

Fig. 3 Fatty acid pattern of plasma lecithin in patient with essential fatty acid deficiency showing decreased levels of $18: 2$ and 20:4 and presence of 20:3 19 before Intralipid and reversal of these abnormalities after Intralipid (reproduced with permission from ref 14 ). 

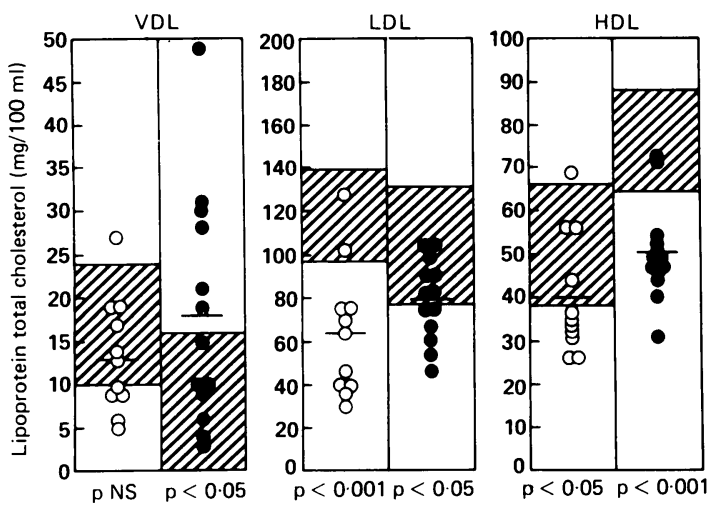

Fig 4 Plasma lipoprotein concentrations in male $(\mathrm{O})$ and female (O) patients with secondary malabsorption. The normal range is hatched. To convert $\mathrm{mg} / \mathrm{dl}$ of cholesterol to mmolll $\div$ by $38 \cdot 7$. Data taken from ref 15 .

\section{Therapeutically induced malabsorption}

OF CHOLESTEROL, BY NEOMYCIN

It is now time to consider the other side of the coin namely, the various ways in which malabsorption can be deliberately induced in order to decrease serum lipids in patients with hyperlipidaemia. One means of doing this is by administering neomycin in a dose of 1-2 $\mathrm{g}$ a day. The numerous amino groups on neomycin make it highly cationic and when added in vitro to a solution of mixed micelles, which have a net negative charge, precipitation occurs due to ionic interaction. ${ }^{18}$ Neomycin precipitates virtually all the fatty acid, monoglyceride and cholesterol present in mixed micelles but only a small proportion of the taurocholate.

Neomycin was introduced as a cholesterol lowering agent by Samuel who used it to treat patients with heterozygous familial hypercholesterolaemia. Studies in patients given a test meal followed by aspiration and ultracentrifugation of the duodenal contents showed that most of the cholesterol, bile acid and fatty acid was present in the micellar phase. When neomycin was given at the same time as the test meal, however, there was a very significant increase in the amount of cholesterol and fatty acid in the precipitate. $^{14}$

The functional consequences of this action were assessed by measuring the faecal excretion of fat and cholesterol off and on neomycin. The percentage of a dose of labelled cholesterol and the amount of fat excreted were both increased by neomycin administration, indicating reduced absorption. The small dose of neomycin used was insufficient to cause mucosal damage, nor was the effect of neomycin

dependent upon its antibiotic properties, as was confirmed in germ free pigs..$^{20}$

OF BILE ACIDS, BY ANION-EXCHANGE RESINS

AND PARTIAL ILEAL BYPASS

Another drug which induces malabsorption, although it affects bile acids rather than cholesterol, is the anion exchange resin cholestyramine. A marked increase in bile acid excretion occurs in subjects given cholestyramine but an even greater increase in excretion occurs after the creation of a partial ileal bypass. This operation, which was invented by Buchwald, involves transecting the ileum at the junction of the distal and middle one-third and then bypassing the distal one-third or $200 \mathrm{~cm}$ by anastomosing the proximal cut end to the caecum.

Spengel et $\mathrm{al}^{21}$ compared the effects of cholestyramine $16 \mathrm{~g}$ per day, and partial ileal bypass in a small group of patients with familial hypercholesterolaemia. Partial ileal bypass was more effective than cholestyramine in reducing LDL cholesterol levels in these patients. Low density lipoprotein turnover studies showed that the reduction in LDL cholesterol was the result of an increased rate of LDL catabolism, which was more marked after partial ileal bypass than during cholestyramine therapy. There was a strong inverse correlation between the concentration of LDL cholesterol and the fractional catabolic rate of LDL in these patients, the lowest concentrations of LDL cholesterol and the highest fractional catabolic rates occurring after partial ileal bypass. The latter procedure was shown to specifically enhance receptor mediated LDL catabolism, as also does cholestyramine.

Cholesterol for bile acid synthesis is partly derived from endogenous synthesis and partly derived from the influx of LDL cholesterol from plasma, mediated by hepatic LDL receptors. Newly synthesised bile acids are secreted in bile and then mainly reabsorbed in the terminal ileum, only a small proportion being excreted in the faeces. In patients with heterozygous familial hypercholesterolaemia, who have fewer hepatic LDL receptors than normal,22 a relatively greater proportion of bile acids is presumably derived from endogenous cholesterol synthesis, although reabsorption and excretion of bile acids occurs normally. When such a patient is placed on cholestyramine this results in decreased reabsorption of bile acids and an increase in faecal excretion. This provides a stimulus to bile acid synthesis and creates an increased demand for cholesterol which is met both from endogenous synthesis and also by stimulation of hepatic LDL receptors; this results in an increased influx of LDL from blood into the liver. After partial ileal bypass, malabsorption of bile acids is even more marked, causing greater stimulation of 
bile acid synthesis and thus greater stimulation of LDL receptors. The tendency for LDL concentrations to rise subsequently reflects a compensatory increase in endogenous cholesterol synthesis which can be suppressed by administration of the HMG CoA reductase inhibitor, mevinolin,,$^{3.3}$ now called lovastatin. The combination of the latter drug with cholestyramine or partial ileal bypass provides a highly effective means of treating hypercholesterolaemia.

\section{MRC Lipoprotein Team, Hammersmith Hospital, Ducane Road, London W12}

\section{References}

1 MacMahon MT, Thompson GR. Comparison of the absorption of a polar lipid, oleic acid, and a non-polar lipid, $\alpha$-tocopherol from mixed micellar solutions and emulsions. Eur J Clin Invest 1970; 1: 161-6.

2 Sabesin SM, Frase S. Electron microscopic studies of the assembly, intracellular transport, and secretion of chylomicrons by rat intestine. J Lipid Res 1977; 18: 496-511.

3 Thompson GR, Ockner RK, Isselbacher KH. Effect of mixed micellar lipid on the absorption of cholesterol and vitamin $\mathrm{D}_{2}$ into lymph. J Clin Invest 1969; 48: 87-95.

4 MacMahon MT, Neale G, Thompson GR. Lymphatic and portal venous transport of $\alpha$-tocopherol and cholesterol. Eur J Clin Invest 1971; 1: 288.

5 Green PHR, Glickman RM. Intestinal lipoprotein metabolism. J Lipid Res 1981; 22: 1153-73.

$6 \mathrm{Wu}$ A-L, Windmueller H. Relative contributions by liver and intestine to individual plasma apolipoproteins in the rat. $J$ Biol Chem 1979; 254: 7316-22.

7 MacMahon MT, Neale G. The absorption of $\alpha$-tocopherol in control subjects and in patients with intestinal malabsorption. Clin Sci 1970; 38: 197-210.

8 Thompson GR, Lewis B, Booth CC. Absorption of vitamin $\mathrm{D}_{2}{ }^{-} \mathrm{H}$ in control subjects and patients with intestinal malabsorption. J Clin Invest 1966; 45: 94-102.

9 Thompson GR, Lewis B, Booth CC. Vitamin D absorption after partial gastrectomy. Lancet 1966; i: 457-8.

10 Thompson GR. Vitamin D deficiency after gastrectomy.
In: The scientific basis of medicine annual reviews, British Postgraduate Medical Federation. University of London, Athlone Press, 1970): 260-75.

11 Clino-pathological Conference. A case of osteomalacia, osteoporosis and hypercalcacmia. Br Med J 1967; i: 219-23.

12 Thompson WG, Thompson GR. Effect of cholestyramine on the absorption of vitamin $\mathrm{D}_{2}$ and calcium. Gut 1969; 10: 717-22.

13 Shimoyama T, Kikuchi H. Press M. Thompson GR. Fatty acid composition of plasma lipoprotein in control subjects and in patients with malabsorption. Gut 1973: 14: 716-22.

14 Press M. Kikuchi H. Shimoyama T, Thompson GR. Diagnosis and treatment of essential fatty acid deficiency in man. Br Med J 1974; ii: 247-50.

15 Thompson GR, Miller JP. Plasma lipid and lipoprotein abnormalitics in patients with malabsorption. Clin $S_{\mathrm{C}}$ Molec Med 1973; 45: 583-92.

16 Press M, Thompson GR. Plasma post-heparin lipolytic activity in patients with malabsorption: effect of intravenous fat administration. Clin Sci Molec Med 1974; 46: 743-51.

17 Miller JP. Thompson GR. Plasma cholesterol esterification in patients with secondary hypocholesterolaemia. Eur J Clin Invest 1973; 3: 401-6.

18 Thompson GR. MacMahon M. Clacs P. Precipitation by neomycin compounds of fatty acid and cholesterol from mixed micelles. Eur J Clin Invest 1970: 1: 40-7.

19 Thompson GR, Barrowman J, Guticrrez L, Dowling $\mathrm{RH}$. Action of neomycin on the intraluminal phase of lipid absorption. J Clin Invest 1971; 50: 319-43.

20) Thompson GR, Henry K, Edington N, Trexler PC. Effect of neomycin on cholesterol metabolism in the germ-free pig. Eur J Clin Invest 1972; 2: 365-71.

21 Spengel FA, Jadhav A, Duffield RGM, Wood CB, Thompson GR. Superiority of partial ileal bypass over cholestyramine in reducing cholesterol in familial hypercholesterolaemia. Lancet 1981; ii: 768-70.

22 Harders-Spengel K. Wood CB. Thompson GR, Myant NB, Soutar AK. Differencees in saturable binding of low density lipoprotein to liver membranes from normocholesterolemic subjects and patients with heterozygous familial hypercholesterolemia. Proc Natl Acad Sci USA 1982; 79: 6355-9.

23 Thompson GR, Ford J, Jenkinson M. Trayner I. Efficacy of mevinolin as adjuvant therapy for refractory familial hypercholesterolaemia. $Q J$ Med 1986: new series: 60: $803-11$. 